\title{
Category Change in the Absence of Cognitive Conflict
}

Ramsburg, J. T., \& Ohlsson, S

\section{Category Change in the Absence of Cognitive Conflict}

Learning that adds new knowledge to the learner's memory with little or no change in prior knowledge is monotonic. The learner's knowledge base grows steadily. If the learner has little prior knowledge of the relevant subject matter, this view serves as a first approximation. But when the learner already has some knowledge of the relevant subject matter and, moreover, new information contradicts that prior knowledge, then learning might involve complicated interactions between the two (Chinn \& Brewer, 1993). If the learner must override or reject his or her prior knowledge, his or her knowledge base shrinks as well as grows, so learning is nonmonotonic (Ohlsson, 2011). Cognitive change of this latter sort is variously referred to as attitude change, belief revision, conceptual change, restructuring, theory change, and deep learning (Ohlsson, 2011). In this article, we adhere to the usage that has become common in the learning sciences and call it conceptual change.

There are multiple theories of the cognitive processes involved in conceptual change. Özdemir and Clark (2007) distinguish between knowledge-as-theory perspectives and knowledge-as-elements perspectives. Theories in the former category conceptualize intuitive and informal knowledge as theory-like in character, and the process of change consequently shares structural features with theory change in science (Kitcher, 1993; Kuhn, 1970; Lakatos, 1980; Nersessian, 2008; Popper, 1959/1972; Thagard,, 1992; Toulmin, 1972). In an influential formulation, Hewson and Hewson (1984), Posner et al. (1982), and Strike and Posner (1982) proposed that students must be dissatisfied with their current conception before they are ready to acquire a new conception. Dissatisfaction, in turn, is caused by the accumulation of anomalies, that is, situations in which the current conception does not lead to successful explanations or problem solutions.

Subsequent theories have offered descriptions of the processes and mechanisms by which the change is executed. For example, the categorical shift theory describes conceptual change as a process that re-classifies a phenomenon or event under a different ontological category when the current classification turns out to be unworkable (Chi, 2005, 2008; Chi \& Brem, 2009; Chi \& Hausmann, 2003; Chi \& Roscoe, 2002; Slotta \& Chi, 2006; Chi, Slotta, \& de Leeuw, 1994; Slotta, Chi, \& Joram, 1995). Vosnidaou and co-workers have proposed that conceptual change involves the construction and revision of mental models. New information can be distorted in the process of being integrated into an existing mental model (Vosniadou, 1994; Vosniadou \& Brewer, 1992; Vosniadou \& Skopeliti, 2013).

In developmental psychology, Piaget (1985/1975) proposed that disequilibrium -- a lack of balance between assimilation and accommodation -- is the driving force in cognitive development. Also in a developmental context, Carey (2009) has described conceptual change in terms of the three processes of coalescence, differentiation, and Quinian bootstrapping. The latter process uses external symbols (words, etc.) as placeholders for concepts that are not yet fully mastered. The symbols gradually accrue meaning through a variety of cognitive mechanisms and processes. These processes are triggered when "children notice failed predictions and internal contradictions among their beliefs" (p. 415). The latter point has been stated more emphatically by proponents of the theory-theory (Gopnik \& Wellman, 1994). "Theories may turn out to be inconsistent with the evidence, and because of this theories change" (Gopnik \& Meltzoff, 1997, p. 39). Although other processes are involved as well, the processing of counterevidence is the 
most important: "Theories change as a result of a number of different epistemological processes. One particularly critical factor is the accumulation of counterevidence to the theory" (Gopnik \& Meltzoff, 1997, p. 39).

To summarize, the multiple knowledge-as-theory perspectives hypothesize different conceptual change processes, but they share the principle that those processes are triggered by negative cognitive outcomes. The latter include direct contradiction or refutation by someone else (e.g., teacher, text, etc.); inability to explain a phenomenon; mismatches between current beliefs and direct observation; unfulfilled expectations; failure to solve a relevant problem; and so on. In general, the difficulty in making a conception 'work' is the main condition that triggers the search for, or the construction of, a new conception. Although there are subtle distinctions among these different types of outcomes, for present purposes we will refer to them as instances of cognitive conflict, contradiction, or falsification, using these terms interchangeably.

The principle that non-monotonic cognitive change is triggered by cognitive conflict has high a priori plausibility. Without falsification or contradiction, a person would presumably lack motivation or reason to change. Why would the cognitive system override, revise, or replace its current knowledge, unless there is evidence that it is erroneous or incomplete? Cognitive economy dictates that a person does not try to fix a knowledge base or belief system that isn't broken. Only after the person has encountered some kind of difficulty in applying his or her current conception is it rational for him or her to search for or construct an alternative conception.

The hypothesis that cognitive conflict is a prerequisite for non-monotonic change predicts that weak learning gains can be strengthened by deliberately inducing cognitive conflict instead of waiting for such conflicts to occur spontaneously. There are various interventions that can serve to induce a cognitive conflict. In the simplest case, the instructor informs the student that his or her current conception is mistaken or incorrect, via either spoken discourse or a refutation text (Sinatra \& Broughton, 2011; Ariasi \& Mason, 2014). Alternatively, the instructor can demonstrate that the prior conception fails to account for relevant evidence or to predict the outcome of a laboratory demonstration (Lee \& Byun, 2012; Lin, 2007). Yet other methods for inducing cognitive conflict have been tried, including animations and simulations (Çalik, Kolomuç \& Karagölge, 2010; Dega, Kriek \& Mogese, 2013).

Numerous studies have investigated the effects of cognitive conflict interventions. In a common approach, the researcher selects some instructional scenario in which learning is known to be weak, adds a cognitive conflict component, and measures whether learning improves, either quantitatively or qualitatively, compared to a control condition that delivers the same instruction without any conflict intervention. After reviewing a decade of research, Strike and Posner's (1992) wrote: "One of the most important findings of the misconception literature...is that misconceptions are highly resistant to change" (p. 153). Research on the cognitive conflict strategy was reviewed again a decade later by Limón (2001). She found that although there are cases in which inducing a cognitive conflict produces positive benefits, those benefits are typically smaller than expected or desired, and in the majority of studies there is no measurable benefit from the cognitive conflict intervention. She wrote that "...the most outstanding result of the studies using the cognitive conflict strategy is the lack of efficacy for students to achieve a strong restructuring and, consequently, a deep understanding of the new information" (p. 364). Subsequent research has confirmed that although cognitive conflict interventions can work in some learning scenarios (Başer, 2006), the effects of such interventions are typically weak when compared to other types of instruction (Zohar \& Aharon-Kravetsky, 2005; Heddy \& Sinatra, 
2013).

Since Limon's (2001) review, cognitive change researchers have investigated the influence on conceptual change of a broader range of cognitive and non-cognitive factors, including affect and motivation, individual differences, metacognition, epistemological beliefs, and intention to change (Cordova et al., 2014; Heddy \& Sinatra, 2013; Kang et al., 2010; Sinatra \& Pintrich, 2003; Trevors \& Muis, 2014; Zohar \& Aharon-Kravetsky, 2005). As a result, we now have a richer description of the conceptual change process. However, broadening the range of factors considered does not by itself answer the question whether cognitive conflict is necessary to trigger conceptual change. Taken at face value, the relative lack of effect of such conflicts across a broad range of studies falsifies the cognitive conflict hypothesis: The difficulty of conceptual change must reside elsewhere than in conflict, or rather the lack thereof, between misconceptions and normatively correct subject matter.

This conclusion implies that researchers should seek to envision cognitive mechanisms and processes that can accomplish conceptual change even in the absence of cognitive conflict. In what Özdemir and Clark (2007) call knowledge-as-elements theories, knowledge is conceptualized as a loosely organized collection of individual knowledge elements, sometimes called phenomenological primitives, or p-prims (Clark, 2006; DiSessa, 1993; DiSessa, Gillespie, \& Easterly, 2004; DiSessa \& Sherin, 1998; Southerland, Abrams, Cummins \& Anzelmo, 2001). In the course of learning, the p-prims undergo stepwise modifications and revisions such that the collection as a whole morphs towards greater similarity to, and overlap with, the normatively correct subject matter. In this type of theory, cognitive conflict plays at most a minor role. The theoretical difficulty is to understand how multiple minor revisions of local knowledge elements can result in non-monotonic learning. How can many small steps take a person from one conception (e.g., the Earth is flat) to a contradictory conception (e.g., the Earth is spherical)?

In past work, we proposed a conceptual change mechanism called resubsumption (Ohlsson, 2009; 2011, Chap. 10). The purpose of the Resubsumption Theory is to explain how non-monotonic cognitive change might occur in the absence of a cognitive conflict. One core idea of this theory is similar to the ontological category shift proposed by Chi and co-workers: An object, event, or phenomenon subsumed under some concept, schema, or (informal) theory X can be understood differently by being assimilated to, or subsumed under, an alternative concept, schema, or (informal) theory Y. The Resubsumption Theory situates cognitive shifts of this sort within a larger processing context that contains several other key components and principles (Ohlsson, 2009; 2011, Chap. 10).

First, human beings are ubiquitous learners. We are continuously encoding communications and experiences, gradually building up concepts, schemas, and theories in order to make sense of the world. This ubiquitous encoding process, by itself, is typically monotonic. The concepts, schemas, and informal theories it generates are normally consistent with each other, and they are typically activated in order to support cognitive processing within the domains that gave raise to them. Intuitive concepts about, for example, diet and health are normally activated in connection with food purchases, discussions about how to stay healthy, etc.

A second key idea in the Resubsumption Theory is that concepts, schemas, and theories that are constructed in order to make sense of one domain of experience or discourse have the potential to apply to some other domain as well, even if the learner was not considering that other domain at the time the knowledge structures were originally constructed. For example, the principle of one person, one vote as it applies to the domain of electoral politics might turn out to apply to computer science: A recommender system might produce judgments by tallying the 
'votes' of large number of independent modules, as an alternative to an algorithmic or analytical solution. As a second example, the principle of natural selection, once discovered in the context of evolutionary biology, might turn out to be applicable to economic markets, the human immune system, as well as other systems.

The possibility of multiple, unanticipated applications of knowledge structures created within one domain to other domains creates the possibility that a person finds himself or herself being able to think about some domain in two different ways. Because the two concepts, schemas, or informal theories both apply to one and the same domain, they are likely to be activated in parallel and compete for control of action or discourse pertaining to that domain. Response competition is likely to be resolved by the accumulation of evidence that one knowledge structure or way of thinking has a higher cognitive utility than the other; that is, it leads more often to successful problem solutions, correct answers, convincing arguments, effective goal attainment, correct predictions, and so forth. Over time, the alternative conception might turn out to have consistently higher cognitive utility than the original one, and it becomes the person's normal way of thinking about the relevant domain.

The Resubsumption Theory shares differences and similarities with both the knowledgeas-theory and knowledge-as-elements perspectives. With respect to the nature of knowledge, the resubsumption idea only claims that knowledge representations are abstract in the sense that they are not bound to the domain within which they were created but can be articulated vis-à-vis concrete phenomena from different domains. The theory is agnostic with respect to other aspects of knowledge. Nevertheless, most of the examples used to support the plausibility of the theory are closer to the knowledge-as-theory perspective (Ohlsson, 2009). On the other hand, the basic cognitive processes postulated by the Resubsumption Theory are not logic-like: the Modus Tollens inference rule that underpins falsification is replaced by encoding into memory, retrieval through spread of activation, response competition based on cognitive utility (not evidence for veridicality); and so on. Crucially, cognitive conflicts are different in character and play a different role in resubsumption than in the knowledge-as-theory view. Conflicts are not logical contradictions but competitions among knowledge structures for the opportunity to apply (Ohlsson, 2013). Dissatisfaction with the prior conception of a domain D plays no role. The shift to an alternative theory for $\mathrm{D}$ is driven by the strengths and successes of the alternative theory, not by the dissatisfactions or problematic outcomes associated with the person's prior conception of D. In short, the resubsumption perspective differs in certain respects from both the knowledge-as-theory and knowledge-as-elements perspectives.

It would be premature to take the weak and variable effects of conceptual conflict interventions as sufficient reason to reject the knowledge-as-theory perspective and adopt either the knowledge-as-elements or the resubsumption perspective. As Limón (2001) pointed out, successful learning of real subject matter in real instructional scenarios depends on multiple factors. "...many of the difficulties found in the application of the cognitive conflict strategy in the classroom are closely related to the complexity of factors intervening in the context of school learning" (Limon, 2001, pp. 364-365). The latter include motivation, learning strategies, epistemological beliefs, attitudes, teacher strategies, teacher training, and so on. None of these factors is sufficient by itself to guarantee successful learning, but weak learning will result if any one factor is absent or not implemented in a felicitous way. If weak learning is due to some other factor than the lack of cognitive conflict, then adding a conflict intervention while keeping the rest of the learning scenario constant should not be expected to improve learning. The problem of multiple determinants of learning outcomes is the more intractable, the more complex the 
learning scenario, so it makes some ecologically valid classroom studies difficult to interpret.

The problem of multiple factors is made yet more intractable when researchers choose to study learning scenarios in which learning is known to be weak or unsuccessful, as is the case for many school science topics. This is a natural choice when the goal is to identify interventions that facilitate or improve learning in real classrooms and with real subject matters. However, the focus on instructional scenarios that have weak learning outcomes complicates the interpretation of the effect of cognitive conflict interventions. Suppose that cognitive conflict is in fact necessary for conceptual change. Then the absence of conflict predicts weak or non-existent learning. But if some other factor in the relevant learning scenario is also non-felicitous, then the weak learning has two causes. In this case, adding a conflict intervention is unlikely to improve learning (Replacing a dead battery in a flashlight does not make it shine if the bulb is burned out as well.). In short, in scenarios in which learning is unsuccessful and learning outcomes are determined by multiple factors, a weak effect of a conflict intervention is compatible with both the hypothesis that cognitive conflict is necessary and its opposite.

One response to this problem is to study simple situations in which there are fewer factors that determine the outcome, and those factors can be identified, measured, or controlled. In addition, basic research studies should focus on situations in which learning with conflict is successful. Successful learning shows that the factors that determine the outcome in that scenario are in fact implemented in a felicitous way. The question then becomes what happens when the cognitive conflict is deleted from the instructional sequence. If learning is significantly slowed down or less accurate, we can conclude that the lack of cognitive conflict must be the cause, so conflict is necessary after all. If learning is unaffected, we can conclude that the conflict is not a necessary condition for successful learning.

Another methodological challenge highlighted by Limón (2001) is to implement an accurate manipulation check: To interpret the outcome of a study in terms of the effects, or the lack thereof, of a cognitive conflict, we need to know whether the learners did in fact experience such a conflict.

Common sense holds that people are prone to ignore or reject contradictory information, or to process it in such a way as to minimize its impact. This commonplace observation is confirmed by several lines of systematic research, including research on attitude change (Maio \& Haddock, 2007), belief revision (Taylor \& Ahn, 2012; Walsh \& Johnson-Laird, 2009), cognitive dissonance (Harmon-Jones \& Mills, 1999), and confirmation bias (Nickerson, 1998). Consistent with what has been found in these areas, Chan et al. (1997) wrote, “...even when students are confronted with contradictory information, they are often unable to achieve meaningful conflict or to become dissatisfied with their prior conceptions" (p. 2). Indeed, the tendency to sweep new information under the cognitive rug is so strong that even scientists are prone to it (Barber, 1961; Chia, 1998).

If students are not aware of the intended conflict because they are too good at ignoring the contradictory information, then the weak effects of the cognitive conflict interventions do not support any strong conclusions; perhaps conceptual change would be accelerated if the students did notice the conflict. One remedy for this problem is to study simple subject matters or learning scenarios in which the contradictory information is so simple, short, and clear that it is highly unlikely that the learner fails to notice it.

Finally, we resonate with Limon's (2001) concern about the measures used to assess the effect of cognitive conflict interventions. Recent experimental studies provide strong support for the hypothesis that prior conceptions do not disappear from memory, even when learning is 
successful. Reaction time studies show that misconceptions can continue to influence cognitive processing, even in adults and experts (Babai \& Amsterdamer, 2008; Babai, Sekal \& Stavy, 2010; Potvin et al., 2015; Shtulman \& Valcarcel, 2012). This finding implies that the key process engaged by a post-test knowledge assessment item is response competition: A question or problem is likely to activate both the prior conception and the newly learned conception, and which conception gets to control the student's response is a matter of the relative strengths of the relevant retrieval paths. Given that an entrenched conception is likely to have accrued activation over a long time, while a newly learned conception has no such track record, it is to be expected that the prior conception wins out, at least sometimes, resulting in a misconception response that gives the impression that learning failed or had only weak effect. That is, misconceptions that are deeply rooted in everyday life might dominate a student's responses to posttest items even when the more correct conception has in fact been successfully encoded into memory.

One response to this difficulty is to study the learning of subject matters that are not deeply rooted in real life. In addition, we need forms of assessment that allow us to track the student's tendency to rely on the misconception versus the newly learned conception independently of each other. If dissatisfaction with a current conception is a prerequisite for learning a new conception, then these two dimensions of the learners' behavior should be temporally displaced with respect to each other. Unlearning should precede relearning. That is, reliance on the prior conception should decrease significantly before reliance on the new conception starts to rise.

To summarize, the bulk of the evidence shows a pattern of weak or non-existent benefits of adding cognitive conflict interventions to learning scenarios in which conceptual learning is less successful than desired. This finding is compatible with the idea that cognitive conflicts are not, after all, necessary for conceptual change. However, learning is a function of multiple factors, so the observed outcome is also compatible with the hypothesis that there is in fact a need for conflict, but that successful learning is blocked by some other non-optimal or infelicitous factor. The difficulties of interpretation is due in part to the (from many points of view very reasonable) choice of studying complex, realistic subject matters for which learning outcomes are determined by many different factors. The problem is acerbated by the (equally reasonable) focus on situations in which learning is known to be weak or unsuccessful. In that situation, a positive outcome is interpretable but a negative outcome is not. In addition, it is difficult to design a manipulation check that will reveal whether the learners experienced any conflict. It is also difficult to design a post-test assessment that will activate or engage the newly learned conception while leaving the prior conception dormant (even if the latter is still available in memory). In the ideal case, the assessment should allow us to track the unlearning of the prior conception and the learning of the new conception over the course of learning and independently of each other. Taken together, these methodological difficulties prevent the body of work on cognitive conflict interventions to decisively support or falsify the hypothesis that cognitive conflict is needed to trigger non-monotonic change.

The purpose of the present work is to contribute to the expanding empirical basis for evaluating different perspectives on the role of cognitive conflicts in non-monotonic cognitive change. In particular, we address directly the question whether cognitive conflict in the sense of falsification is a necessary prerequisite for a non-monotonic change. We report three experimental studies that demonstrate the psychological reality of successful, non-monotonic learning in the absence of falsifying or contradictory information. The studies show that contradictory information is not a prerequisite for learning in a category learning scenario that 
we refer to as re-categorization (Cosejo, Oesterreich \& Ohlsson, 2009; Ohlsson \& Cosejo, 2014). In this scenario, the participants learn to categorize a novel set of stimuli through the standard procedure used in categorization experiments (Ashby \& Maddox, 2005): The participants view a potential category member; judge whether it is a member; receive feedback on their judgment; and go on to the next trial; the process continues until the learner passes some criterion of mastery.

In our experiments, once the participants show that they have mastered the category, the category definition is changed without warning. To continue to classify correctly, the learner has to un-learn the initial definition of the category, change his or her definition of the category, and, consequently, begin to categorize the relevant stimuli in a way that contradicts the initially learned category. The roots of the re-categorization paradigm stretch back to work on reversal and nonreversal shifts in the 1950s and 60s (e.g., Kendler \& D'Amato, 1955), and the use of the Wisconsin Card Sorting Task (WCST) to assess perseveration in clinical work (Berg, 1948), but it is more closely related to recent experimental work on category learning (Doumas \& Hummel, 2013; Ecker et al., 2011; Sewell \& Lewandowsky, 2011; Taylor \& Ahn, 2012). Our stimuli (the potential category members) are more complex and designed to evoke a science learning scenario, and we analyze our data differently. Some descriptive features of behavior in the recategorization paradigm were reported in Ohlsson and Cosejo (2014).

The advantages of the re-categorization paradigm for present purposes include experimental control over the participants' prior knowledge, the ability to collect temporally dense and fine grained data, the simplicity of the subject matter, and the ability to control the content of the feedback messages given to the participants (Cosejo, Oesterreich, \& Ohlsson, 2009; Ohlsson \& Cosejo, 2014). Categorization of artificial and hence previously unknown objects is a simple learning situation in which the instances - the potential category members seen during learning and the content, accuracy, and frequency of the feedback are the main determinant of learning outcomes. We can easily design learning tasks for which learning is successful by carefully moderating the complexity of the initial category, the switch, and the target category. Cognitive conflicts are straightforward and impossible to overlook, consisting of a simple choice (is an item a category member or not?) immediately followed by feedback about the membership status of that very instance. Also, posttest items can probe the learner's state of knowledge after completing the learning task: Are his or her categorization responses to previously unseen instances guided more by the initially learned category, or more by the target category?

We created two main learning conditions within the re-categorization paradigm, one in which falsifying information was available and one in which it was not. Our participants were able to learn under both conditions, and the question is what happens when we delete the possibility of falsification. If falsifying information is essential, the participants in the former condition should learn faster and/or more accurately than those in the latter condition. To preview our main result, we found no measurable effect of access to falsifying information in the rate and quality of non-monotonic category change across three experiments.

\section{EXPERIMENT 1}

The re-categorization procedure used in this study presented images (supposedly electron microscope photos) of fictitious alien bacteria. These were to be categorized with respect to their resistance to atmospheric oxygen; see details in the Method section. All participants were given both confirming and falsifying feedback on their categorization judgments. We refer to this phase as initial learning, and the category definition learned as the initial category (a.k.a. the 
'misconception'). The conditions of initial learning were identical for the two experimental groups.

After the initial learning phase, the category definition was changed. The participants were not informed of the change, and there was no break in the experimental procedure. The procedure continued with feedback now being based on the new category definition. We refer to this second phase of the experiment as target learning, and the new category definition acquired in this phase as the target category.

During target learning, the participants were exposed to one of two feedback conditions. The participants in the Complete condition continued in the same way as during initial learning. That is, they viewed both instances and non-instances of the target category, so they received both confirmatory feedback (when they categorized correctly) and also directly falsifying feedback (when they categorized incorrectly).

The participants in the second, Confirmation-Only condition were presented with images that had been altered in such a way that the initial category could not be directly falsified. This was accomplished by deleting crucial elements from the images. For example, if category membership was dependent on the shape of the 'ribosomes' visible in the images, then the participant might be shown an image in which the 'ribosomes' were missing, so their shape was unknown; see the Method section for details. The deleted parts carried the crucial information about category membership according to the initial (but not the target) category definition. Hence, participants in this group did not encounter any information that enabled them to falsify their initial category, but otherwise they received the same information as the participants in the Complete condition. In particular, they saw as many instances of the target category as the participants in the Complete condition, and they received the same feedback.

In short, the purpose of Experiment 1 was to compare re-categorization, a simple case of non-monotonic conceptual learning, in the presence and absence of falsifying feedback vis-à-vis an initial category. The hypothesis that negative cognitive outcomes such as falsification and contradiction are prerequisites for non-monotonic learning predicts that the participants in the Complete condition learn faster and more accurately than the participants in the ConfirmationOnly condition. An alternative outcome is that contradictions disrupts learning, in which case the participants in the Confirmation-Only condition will learn faster or better than those in the Complete condition. An alternative outcome is that contradictions neither trigger nor disrupt non-monotonic category learning, in which case there is no reason to expect one of these conditions to learn the target category faster or more accurately than the other.

A second hypothesis pertains to the temporal sequence of un-learning and re-learning: If dissatisfaction with a current category is a prerequisite for non-monotonic category change, then reliance on the initial category should begin to decrease significantly before reliance on the target category begins to increase. In contrast, if the un-learning of the initial category definition is unrelated to the learning of the target category, the two processes can occur in parallel, neither waiting for the other.

A third research question pertains to the final knowledge state of the participants who successfully acquire the target category. One interpretation of the cognitive conflict hypothesis is that the new conception replaces the prior conception. In this view, the prior conception is deleted from memory in the course of learning. In this case, participants' ratings of category features collected after completed learning should only reflect the new conception. However, an alternative possibility is that the new conception is added to memory without changes in the prior conception. In this case, posttest ratings might be influenced by both conceptions. 


\section{Participants}

\section{Method}

One hundred twenty psychology students participated in the study for course credit. The age of the participants in the subject pool ranges from 18 years old and older. The great majority of participants are between 20 and 35 years old. The gender balance is approximately $60 \%$ female. Participants were seen in groups ranging from 2 to 12 participants per group. The groups were randomly assigned to conditions, which yielded 66 participants in the Complete condition and 54 participants in the Confirmatory-Only condition.

Materials

The materials consisted of images of 128 fictional bacteria (see Figure 1). The bacteria have six different parts each of which varies along one binary attribute, resulting in 64 complete variants: Nuclei (grey or black), Headbulbs (three or none), Ribosomes (bent or straight), Tail Cilia (present or absent), Cell Membrane (singular or double), and Cytoplasm (white or grey). In addition, there were some variants which were incomplete; that is, they lacked one of the parts and hence did not provide any information regarding its value on the associated attribute. For example, some images did not show the nuclei and some did not show the tail (see Figure 2).

Insert Figure 1 about here

The images were presented on a computer screen using the E-Prime experiment control software; see (www.pstnet.com/products/E-Prime/default/).

Insert Figure 2 about here

\section{Measures}

Importance ratings. Participants rated the importance of each feature of the bacteria (e.g., "How important is/was having a clear cytoplasm") on a 7-point Likert scale from 1 (Not at all) to 7 (Extremely). The ratings scale was administered twice, once before the learning task and again at the end of the experiment.

Percentage correct. Each training block consisted of 16 trials. The proportion of correct responses was computed for each training block and each participant, and averaged across participants.

Mastery criterion. If a participant responded correctly to at least 14 of 16 trials within a training block then he or she was considered to have learned the category. This mastery criterion was applied in each phase of training, initial category learning and target category learning. A participant needed to learn the initial category in order to be included in target learning analyses.

Target or misconception consistent responses. In addition to the percentage of correct answers, we analyzed the categorization responses to track the participants' reliance on the initial and target categories. We classified each response as consistent with the misconception, consistent with the target conception, or neither. Consistent with the misconception means that the stimulus variant displayed the feature that determined category membership, and the participant judged that variant as a category member. For those items presented in the Confirmation-Only condition that were incomplete, the misconception feature was unknown, so responses were classified as misconception consistent when the target was present and the 
participants judged the variant as not oxygen resistant. Participants had the option to conclude that they didn't know if a variant was oxygen resistant or not. Similarly, consistent with the target conception means that the stimulus displayed the target feature, and the participant judged it as oxygen resistant. The proportion of responses that were consistent with either conception was computed for each training block and each participant and averaged across participants. Design

The study was a between-participants design with two conditions (Complete and Confirmatory-Only). Both groups first learned the initial category definition. The materials and procedure were identical for both groups in the initial learning phase. After the initial learning, the correct category definition was changed. The participants were not informed of the change. In the second phase, the participants received training on the target category. The two target training conditions differed in the stimuli presented and hence in the information provided; see next few paragraphs for details.

Phase 1: Initial learning. Participants first learned to categorize whether an alien bacterium was oxygen resistant based on feedback that was consistent with the misconception feature (black nuclei). The participants received five training blocks of 16 trials each. Each training block was balanced to include in randomized order six images that contained the misconception feature, six images that contained the target feature, two images that contained neither, and two images that contained both the misconception and the target. (The purpose of the latter was to make the learning situation somewhat more challenging by introducing a small amount of noise into the information the participants received.). After five training blocks, the initial training phase was terminated and the next phase began.

Phase 2: Target learning. After the end of the initial learning phase, the feature that determines oxygen resistance changed to bent ribosomes (the target feature). The participants were not informed that the category had changed. Participants had five training blocks of 16 randomized trials to learn that bent ribosomes determined oxygen resistance. The target training occurred under two different conditions that differed in the kinds of images presented.

Condition 1: Complete Stimuli. The stimuli presented in this condition consisted of images that were similar to what participants had already seen during initial learning. Each training block was balanced to include, in randomized order, six images that contained the misconception feature, six images that contained the target feature, two images that contained neither, and two images that contained both the misconception and the target features

All parts of the bacteria were visible on the screen to enable participants to falsify their prior category definition. For example, in Phase 1, the participant learned that black nuclei were responsible for oxygen resistance. In Phase 2, the participant was confronted with an image containing black nuclei with feedback stating that the bacterium was not oxygen resistant. This feedback provided an opportunity for the learner to directly falsify the prior conception. Similarly, when the learner was confronted with an image that did not have a black nucleus, but was said in the feedback message to be oxygen resistant, he or she had an opportunity to conclude that another part of the bacterium must be responsible for oxygen resistance.

Condition 2: Confirmation-Only Stimuli. In this condition, the participants were not shown any stimuli that could be used to directly falsify the misconception. Some images that would have been category members if they had been complete did not include the nuclei, resulting in an inability of the learner to directly falsify the initial category definition learned in Phase 1. Each training block was balanced to include in randomized order six images that did not display the nuclei, six images that fit the target category, two images that fit neither category, 
and two images that fit both the misconception and the target categories. The purpose of the latter was to make the learning situation somewhat more challenging by introducing a small amount of noise into the information the participants received. All other aspects of Condition 2 were identical to Condition 1.

\section{Procedure}

Participants were seated in separate cubicles. Each participant first participated in a training session, which consisted of a series of PowerPoint slides outlining how one can sort a variety of objects into different categories. The training session ended with participants categorizing simple stick figures based on their features. When participants finished with the initial training activity, they moved on to the bacteria categorization task.

Participants read the instructions for the task on the computer screen and asked questions if needed. Participants were asked to pretend that alien bacteria were recently discovered on a distant planet and that scientists needed to determine whether some variants of the bacteria were oxygen resistant (and hence could potentially spread in the Earth's atmosphere). The participants were shown a bacterium with its parts labeled; see Figure 1.

Participants were then asked to rate how important each part was in determining oxygen resistance on a 7-point Likert scale from 1 (Not at all) to 7 (Extremely). After rating the features, participants saw a prompt that emphasized the importance of determining which bacteria were oxygen resistant. In each trial, the participant was shown one bacterium image and asked whether the pictured bacterium was oxygen resistant. Participants indicated their responses via the computer keyboard. The enabled responses were: $y$ for "yes, it is oxygen resistant", $n$ for "no, it is not oxygen resistant", and $d$ for "I don't know".

Participants received immediate feedback from the computer. The feedback did not say whether the participant's response was correct or not. Instead, it stated whether the shown bacterium was or was not oxygen resistant. Participants were instructed to make as few errors as possible.

After completing all trials, participants were again asked how motivated they were to perform the task well and to rate the importance of different features in determining oxygen resistance on the same 7-point Likert scale as before. The participants keyed in an open-ended response about which features they thought determined oxygen resistance. On the following screen they were asked whether oxygen resistance was always determined the same way. Finally, the participants answered demographic questions.

\section{Results}

We wanted to examine whether falsification was necessary for adopting a new method of categorization for those participants who succeeded in learning the initial misconception feature, (as opposed to whether falsification is necessary to learn the target category from scratch). Hence, we focused the data analysis on those participants who passed a criterion of mastery of the initial category; the criterion used was that they correctly classified 14 of 16 alien bacteria in any of the initial five training blocks. Thirty-eight participants in the Complete condition and 36 participants in the Confirmatory-Only condition met the mastery criterion. Due to a computer programming error, the feature importance ratings of 21 participants were not recorded. These participants could not be included in the analysis of the importance ratings, but were included in all other analyses.

\section{Importance Ratings}

An analysis of the importance ratings before categorizing the bacteria was conducted using a one-way analysis of variance (ANOVA). This analysis was performed in order to 
examine whether there were any pre-existing differences in how the participants rated the various bacteria attributes before learning. No differences were found between ratings of attributes across participants, $F(5,312)=1.19, p=.316$.

A two-way ANOVA was used to examine whether there were any differences between groups in how they rated the bacteria features at the conclusion of the re-categorization task. There was a main effect for condition, that is, participants in the Complete condition had higher overall ratings than the Confirmation-Only condition, $F(1,306)=6.54, p=.011, \eta_{\mathrm{p}}{ }^{2}=.021$. There was a main effect for attribute, that is, attributes were rated differently, $F(5,306)=28.99$, $p<.001, \eta_{\mathrm{p}}{ }^{2}=.321$. The main effects were qualified by an interaction between condition and attribute, $F(5,306)=3.39, p=.005, \eta_{\mathrm{p}}{ }^{2}=.053$. Post hoc least significant difference (LSD) pairwise comparisons revealed that participants in the Complete condition rated cell walls, $F(1$, $306)=9.11, p=.003, \eta_{\mathrm{p}}{ }^{2}=.029$ cytoplasm, $F(1,306)=6.25, p=.013, \eta_{\mathrm{p}}{ }^{2}=.020$ and headbulbs, $F(1,306)=4.18, p=.042, \eta_{\mathrm{p}}{ }^{2}=.013$ as more important compared to participants in the Confirmation-Only condition. The Confirmation-Only condition rated nuclei as being marginally more important compared to the Complete condition, $F(1,306)=3.43 p=.065, \eta_{\mathrm{p}}{ }^{2}$ $=.011$. There were no differences in ratings for ribosomes or tail cilia between groups, $F$ ' $s<1$ (see Figure 3).

Insert Figure 3 about here

\section{Learning the Misconception}

Our analysis determined whether random assignment was effective at producing equivalent groups. In order to determine whether participants might differ in their ability to learn the misconception, we examined their performance on the first five blocks via a repeated measures ANOVA with Greenhouse-Geisser correction found a main effect for blocks, that is, regardless of condition, participants improved in performance from blocks 1 thru $5, F(2.32$, $166.84)=66.76, p<.001, \eta_{\mathrm{p}}{ }^{2}=.653$. There was no main effect of condition, $F<1, \eta_{\mathrm{p}}{ }^{2}=.008$ nor did groups differ at rate of learning, $F(2.32,166.84)=1.09, n s ., \eta_{\mathrm{p}}{ }^{2}=.307$. These results suggest that the groups were equivalent in their ability to learn the misconception under these two conditions.

\section{Learning the Target}

Our next step was to assess whether the Confirmatory-Only condition learned the target in blocks 6 through 10. Performance of 14 out of 16 or greater on any of the blocks 6 through 10 was rated as successful learning of the target; we found that 29 of $36(80.55 \%)$ participants correctly learned the target category. Whereas, if participants maintained the misconception for all trials they would have resulted in 0 of 36 participants demonstrating that they learned the target.

Using a chi-squared goodness-of-fit test we measured overall target acquisition (i.e., in general did learning occur yes or no) against a more stringent probability (i.e., chance at 50\%). Specifically, the results revealed that the Confirmatory-Only condition's target acquisition was better than chance, $\chi^{2}(36)=13.44, p<.001$. Similar results were found for the Complete condition in which 29 of $38(76.31 \%)$ participants learned the target, $\chi^{2}(36)=9.00, p<.01$.

\section{Differences between Groups for Target Learning}

We examined whether conditions differed in target acquisition via a chi-squared test-ofindependence that showed that the groups did not differ in target acquisition, $\chi^{2}(36)=2.90, p=$ .09. Removing the opportunity to directly falsify the misconception did not significantly impair 
the participants' ability to adopt a new method of categorization.

We also examined potential differences in learning rate based on condition following the switch. That is, we wanted see whether one group learned faster than the other. A repeated measure ANOVA with Greenhouse-Geisser correction was used to determine whether there was a difference in performance following the switch from the misconception to the target for blocks 6 through 10. The analysis revealed a main effect for blocks showing that participants improved with training, $F(2.646,190.477)=75.01, p<.001, \eta_{\mathrm{p}}{ }^{2}=.671$, and a main effect for condition showing that the Confirmatory-Only condition performed better than the Complete condition, $F(1,72)=7.60, p<.01, \eta_{\mathrm{p}}^{2}=.096$. The interaction was significant. The rate of learning was faster for the Confirmatory-Only condition than the Complete condition, $F(2.646,190.477)=$ $5.21, p<.01, \eta_{\mathrm{p}}{ }^{2}=.146$. These results suggest that the participants in the Confirmatory-Only condition unexpectedly learned the target category faster than the participants in the Complete condition, although the difference is small in magnitude (see Table 1).

Insert Table 1 about here

\section{Response Type by Condition}

To throw additional light on the differences between the two learning conditions, we rescored the participants' responses with respect to whether they were consistent with the misconception (MCR) or the target conception (TCR). (These response types are independent from each other because of the don't know response option.) Figure 4 shows the proportion of MCR and TCR responses for each block, averaged across the participants, for both groups. In blocks $1-5$, the proportion of responses in each block that were consistent with the misconception rose, while the proportion of responses in each block that were consistent with the target conception fell. In blocks $6-10$, these changes were reversed. The main observation is that the curves for the two learning conditions differ minimally. In particular, there is no evidence that participants in the Complete condition learned faster than the participants in the Confirmatory-Only condition. In fact, the latter group had a slight advantage.

Insert Figure 4 about here

\section{Discussion of Experiment 1}

The results of Experiment 1 suggest that the Confirmatory-Only condition might initially speed up learning of a new conception in comparison to the Complete condition. We investigated whether participants would adopt a new conception after the switch and whether rate of learning would vary based on condition. We found that the participants in both conditions adopted the new conception. However, participants in the Complete condition learned at a slower rate than those in the Confirmatory-Only condition. It is possible that this difference was due to the need for participants in the Complete condition to make sense of conflicting information. The sense making absorbed cognitive resources that otherwise would have been available for learning the target category, slowing down the re-categorization process. In addition, we observed that the increase in the participants' reliance on the target category was not delayed with respect to the decrease in the participants' reliance on the initial category; instead, both the unlearning and the re-learning proceeded in parallel. Finally, the bacteria ratings showed that the participants recognized the importance of the target attribute, but still maintained belief in the misconception feature. The behavioral data and self-report data show that learners in both conditions effectively 
learned the target category, with the Confirmatory condition switching faster, but that both groups also maintained a sense of importance vis-à-vis the initial category definition. The issue of whether learning was monotonic or non-monotonic rests on the need for learners to disregard their initial category method and adopt the new categorization method. Learners in both conditions could not demonstrate target learning without disregarding misconception learning. Therefore, the learning was non-monotonic.

\section{Experiment 2}

In Experiment 2, we attempted to replicate the findings of Experiment 1 with minor changes in the procedure to address some concerns. First, there was the concern that participants might be viewing the bacteria images that do not show the dark nuclei as being bacteria without nuclei, which would result in a different interpretation of the stimuli by the participants. To counteract this, the instructions were revised to include the statement that "some of the images of the bacteria that you will see may be INCOMPLETE, that is, all bacteria have the 6 parts described, but some parts may not be visible."

The second concern was that the participants might be biased to think that the internal micro-features of the bacteria images (including the 'nuclei' and the 'ribosomes') were irrelevant for oxygen resistance, as compared to external features like head bulbs and cell walls. To counteract any bias in this respect, the instructions were revised to suggest that parts within the cell body may be influential in determining oxygen resistance.

Finally, we tried to reduce the number of participants that are eliminated from the target learning analysis because they failed to learn the initial category. To this aim, we gave the participants a learning aid in the form of a handout that showed an image of the bacteria with parts labeled (as in Figure 1) as well as a list of the possible values of each attribute. This was meant to serve as a working memory aid.

\section{Participants}

\section{Method}

Sixty-one introductory psychology students participated in the study for course credit. The age of the participants in the subject pool ranges from 18 years old and older. The great majority of participants are between 20 and 35 years old. The gender balance is approximately $60 \%$ female. Random assignment yielded 30 participants in the Complete condition and 31 participants in the Confirmatory-Only condition.

\section{Design \& Procedure}

The same procedure as in Experiment 1 was used in Experiment 2, with the addition of the two learning aids (i.e., the hint in the prompt and the handout).

\section{Results}

Twenty-one participants in the Complete condition and 21 participants in the Confirmatory-Only condition met the criterion for inclusion in analyses (i.e., correctly classifying 14 of 16 alien bacteria in any of the first five blocks).

\section{Importance Ratings}

An analysis of the importance ratings before categorizing the bacteria was conducted using a one-way ANOVA. This analysis was performed in order to examine whether there were any pre-existing differences in how the participants rated the various bacteria attributes. No differences were found between ratings of attributes across participants, $F(5,246)=1.88, p=$ .099 .

A two-way ANOVA was used to examine whether there were any differences between groups in how they rated the bacteria at the conclusion of the re-categorization task. There was 
no main effect for condition, that is, participants in the Complete condition and the Confirmation-Only condition did not differ in overall ratings, $F(1,240)=2.24, p=.136, \eta_{\mathrm{p}}{ }^{2}=$ .009. There was a main effect for attribute, that is, attributes were rated differently, $F(5,240)=$ 21.58, $p<.001, \eta_{\mathrm{p}}{ }^{2}=.310$. There was no interaction between condition and attribute, $F(5,240)$ $=0.36, p=.873, \eta_{\mathrm{p}}{ }^{2}=.008$. Pairwise comparisons for attribute type revealed that regardless of condition participants rated nuclei and ribosomes as more important than any other attribute, $p$ 's $<.001$. The other attributes did not differ between each other, $p$ 's $>05$ (see Figure 5).

Insert Figure 5 about here

\section{Learning the Misconception}

Our first analysis sought to determine whether participants might differ in their ability to learn the misconception via a repeated-measures ANOVA in which we examined percentage correct per block for the first five blocks. We found a main effect for blocks, that is, regardless of condition, participants improved in performance from blocks 1 thru 5, $F(4,160)=55.60, p<$ $.001, \eta_{\mathrm{p}}{ }^{2}=.582$. There was no main effect of condition, $F(1,40)=1.69, p=.201, \eta_{\mathrm{p}}{ }^{2}=.041$ nor did groups differ at rate of learning, $F(4,160)=1.19, p=.319, \eta_{\mathrm{p}}{ }^{2}=.029$. These results suggest that the groups were equivalent in ability to learn the misconception.

\section{Learning the Target}

Our next step was to assess whether the Confirmatory-Only condition learned the target in blocks 6 through 10. Performance of 14 out of 16 or greater on any of the blocks 6 through 10 was rated as successful learning of the target. We found that 15 of 21 (71.43\%) participants correctly learned the target category. Using a chi-squared goodness-of-fit test the results revealed that the Confirmatory-Only condition's target acquisition was better than chance, $\chi^{2}(21)=3.86$, $p=.05$. Alternatively, results for the Complete condition in which 14 of $21(66.67 \%)$ participants learned the target, their target acquisition was not better than chance, $\chi^{2}(21)=2.33, p=.127$.

\section{Differences between Groups}

We examined whether conditions differed in learning the target via a chi-squared test-ofindependence that showed that the groups did not differ in learning the target, $\chi^{2}(42)=.11, p=$ .739. This replicates the finding from Experiment 1 that the ability to directly falsify a misconception is not necessary for learning the new conception.

Given the relatively small sample size for Experiment 2 and the likelihood of differences occurring in earlier blocks we opted to conduct a series of t-tests on target learning blocks 6 through 10 instead of a repeated-measure ANOVA, which might fail to differentiate the effect. The results of the t-tests revealed that participants in the confirmation condition performed better than the Complete condition for block $6, t(40)=4.56, p<.001$ and marginally better on block 7 for a one-tailed t-test, $t(40)=1.67, p=.051$. There were no differences between the groups for blocks 8,9 , and $10, t<1$ (see Table 2 ).

Insert Table 2 about here

\section{Statistical Power}

Due to the modest sample size $(\mathrm{N}=42)$, a post hoc power analysis was conducted using G*Power (Faul, Erdfelder, Lang, \& Buchner, 2007) for blocks 6 and 7, respectively. The power analysis can be used to determine whether the study was under powered. The results revealed that statistical power $(1-\beta)$ was .99 on the basis of the input parameters: effect size $(d=1.41), \alpha$ 
$=.05$, two-tailed, and sample size per group for block 6. Statistical power was .52 for block 7

based on the same input parameters aside from effect size $(d=.63)$. There was sufficient statistical power for block 6, but not for block 7. (Power analysis was not needed for Experiments 1 and 3, because they showed a significant effect in the unexpected direction.)

\section{Discussion of Experiment 2}

The results of Experiment 2 replicated the main finding from Experiment 1. The absence of stimuli in which participants can directly falsify actually increases the rate at which learners adopt the target category. The sample size was relatively low for this study, but the power analysis suggests that there was sufficient power to observe the effect for the sixth block, which is arguably the most important re-learning block. Additionally, the increase in the participants' reliance on the target category was not delayed with respect to the decrease in their reliance on the initial category. Finally, the importance ratings were similar to those in Experiment 1, with both participants in both conditions rating both the target and misconception attributes as important.

\section{Experiment 3}

In Experiment 3, we modified the experimental procedure in several ways. The representation of the various stimuli types was changed (see Design \& Procedure for Experiment 3 ). This was done in order to determine whether the effect would remain with different stimuli configuration sequences. In the prior experiments, we included four non-diagnostic trials in each block, which could be interpreted as supporting the misconception. We removed the nondiagnostic trials for the target training phase of the Complete condition. In their place we included two more direct falsification opportunities and two more target induction opportunities. In accord with the cognitive conflict hypothesis, we increased the number of opportunities to create a cognitive conflict. In addition, we examined whether switching the stimuli presentation on the final block would impact learning. This was done in order to examine whether participants would be able to maintain their learning performance with stimuli from the other condition (i.e., transfer). Finally, we wanted to see if the new stimuli configurations would affect bacteria ratings. Specifically, with more falsification opportunities in the Complete condition one might expect that ratings for the nuclei might go down.

\section{Participants}

\section{Method}

One hundred fifteen introductory psychology students participated in the study for course credit. The age of the participants in the subject pool ranges from 18 years old and older. The great majority of participants are between 20 and 35 years old. The gender balance is approximately $60 \%$ female. Random assignment yielded 53 participants in the Complete condition and 62 participants in the Confirmation condition.

\section{Design \& Procedure}

The same procedure was used as in experiment 2 excluding the modification of stimuli presentations for both groups. Specifically, for the Confirmation condition stimuli for blocks 6 through 9 contained 16 trials for each block: eight images in which the target was known, but the misconception was unknown and eight images in which neither the target nor misconception were present. The tenth block contained eight images, in which the misconception was present, but not the target and eight images in which the target was present, but the misconception was unknown. The Complete condition followed the same format, but swapped the stimuli types. That is, blocks 6 through 9 contained stimuli represented in block 10 of the condition and block 10 contained stimuli represented in blocks 6 through 9 of the Confirmation condition. 


\section{Results}

Forty-four participants in the Complete condition and 32 participants in the Confirmation condition met the criterion for inclusion in analyses (i.e., correctly classifying 14 of 16 alien bacteria in any of the first five blocks).

\section{Importance Ratings}

An analysis of the importance ratings before categorizing the bacteria was conducted using a one-way ANOVA. This analysis was performed in order to examine whether there were any pre-existing differences in how the participants rated the various bacteria attributes. A significant effect was found between ratings of attributes across participants, $F(5,455)=6.52, p$ $=.017$. Post hoc tukey HSD analyses revealed that the only significant difference was between cell walls $(\mathrm{M}=5.07, \mathrm{SD}=.17)$ and ribosomes $(\mathrm{M}=4.27, \mathrm{SD}=.17), \mathrm{p}=.018$.

A two-way ANOVA was used to examine whether there were any differences between groups in how they rated the bacteria at the conclusion of the re-categorization task. There was no main effect for condition, that is, participants in the complete condition and the confirmation condition did not differ in overall ratings, $F(1,456)=.153, p=.696, \eta_{\mathrm{p}}{ }^{2}<.001$. There was a main effect for attribute, that is, attributes were rated differently, $F(5,456)=24.88, p<.001, \eta_{\mathrm{p}}{ }^{2}$ $=.219$. There was no interaction between condition and attribute, $F(5,456)=1.39, p=.226, \eta_{\mathrm{p}}{ }^{2}$ $=.015$. Pairwise comparisons for attribute type revealed that regardless of condition participants rated nuclei as more important than any other attribute, $p$ 's $<.001$. Ribosomes were rated as more important than cell walls, $\mathrm{p}=.024$ and any other remaining attribute, $\mathrm{p}$ 's $<.001$. The other attributes did not differ between each other, $p$ 's $>.05$ (see Figure 6).

Insert Figure 6 about here

\section{Learning the Misconception}

Our first analysis sought to determine whether participants might differ in their ability to learn the misconception via a repeated-measures ANOVA in which we examined percentage correct per block for the first five blocks. We found a main effect for blocks, that is, regardless of condition, participants improved in performance from blocks 1 thru 5, $F(4,296)=93.36, p<$ $.001, \eta_{\mathrm{p}}{ }^{2}=.558$. There was no main effect of condition, $F(1,74)=.58, p=.451, \eta_{\mathrm{p}}{ }^{2}=.008$ nor did groups differ at rate of learning, $F(4,296)=1.26, p=.286, \eta_{\mathrm{p}}{ }^{2}=.017$. These results suggest that the groups were equivalent in their ability to learn the misconception.

\section{Learning the Target}

Our next step was to assess whether the Confirmation condition learned the target in blocks 6 through 10. Performance of 14 out of 16 or greater on any of the blocks 6 through 10 was rated as successful learning of the target. We found that 28 of 32 (87.5\%) participants correctly learned the target category. Using a chi-squared goodness-of-fit test the results revealed that the Confirmation condition's target acquisition was better than chance, $\chi^{2}(32)=18, p<$ .001. Additionally, results for the Complete condition in which 42 of 44 (95.45\%) participants learned the target, their target acquisition was also better than chance, $\chi^{2}(44)=36.36, p<.001$.

\section{Differences between Groups}

We examined whether conditions differed in target acquisition via a chi-squared test-ofindependence that showed that the groups did not differ in target acquisition, $\chi^{2}(76)=1.61, p=$ .204. Removing the opportunity to directly falsify the misconception did not significantly impair the participants' ability to adopt a new method of categorization.

We also examined potential differences in learning rate based on condition following the 
switch. That is, we wanted see whether one group learned faster than the other. A repeated measure ANOVA was used to determine whether there was a difference in performance following the switch from the misconception to the target for blocks 6 through 10. The analysis revealed a main effect for blocks showing that participants improved with training, $F(4,296)=$ 45.28, $p<.001, \eta_{\mathrm{p}}{ }^{2}=.380$, and a main effect for condition showing that the Confirmation condition performed better than the Complete condition, $F(1,74)=10.89, p<.001, \eta_{\mathrm{p}}{ }^{2}=.128$. The interaction was significant. The rate of learning was faster for the Confirmation condition than the Complete condition, $F(4,296)=37.89, p<.001, \eta_{\mathrm{p}}{ }^{2}=.339$. These results suggest that the participants in the Confirmatory condition learned the target category faster than the participants in the Complete condition, although the difference is small in magnitude (see Table $3)$.

Insert Table 3 about here

\section{Discussion of Experiment 3}

The results of Experiment 3 were consistent with the results of the previous two experiments. There was no advantage for the Complete condition over the Confirmation condition. The importance ratings showed that both the initial and the target features were seen as more important than the other features at the end of successful learning. These findings are robust across the variations of our experimental procedure. In addition, both groups were able to perform equivalently well on the $10^{\text {th }}$ block, which represented stimuli from the other condition. This suggests that both conditions could adequately transfer their knowledge of the target category to novel stimuli. Moreover, the difference in performance for block 6 appeared to be even more pronounced than in experiments 1 and 2, even though experiment 3 contained more falsification opportunities in block 6 for the complete condition. This finding is inconsistent with the cognitive conflict hypothesis.

\section{Summary}

\section{General Discussion}

Contrary to what the cognitive conflict hypothesis would lead us to expect, we found no evidence across three experiments that opportunities to directly falsify a category definition systematically facilitated the participants' ability to learn a new definition of that category. In Experiment 1, the group of learners who were presented with both confirming and falsifying instances re-learned the target category at a somewhat slower pace than the participants who were presented with confirming instances but no opportunity to falsify the initial category definition. In Experiment 2, performance immediately after the switch was superior for the confirmation condition compared to the complete condition. In Experiment 3, the participants without opportunity to falsify learned the target category faster than a group with such opportunities. In short, removing any direct cognitive conflict from the instructional sequence did not impair target learning, strongly suggesting that cognitive conflict was not a prerequisite for successful acquisition of the target category.

Consistent with this conclusion, there was no temporal displacement between unlearning and re-learning in any of the three experiments. If dissatisfaction with the initial category had been a necessary trigger for attempts to find a new category definition that would work better, then the rise in the participants' reliance on the target category should have been delayed until there had been significant decrease in their reliance on the initial category. But this was not the case. Instead, the two types of change began immediately after the change in the category 
definition, and proceeded simultaneously and in parallel throughout target learning. The participants began acquiring information about the target category even before they had any indication that their current category definition no longer enabled them to correctly classify the images. This pattern was replicated across all three experiments; see Tables 1, 2, and 3.

Finally, the importance ratings failed to conform to the pattern implied by the cognitive conflict hypothesis. Because the potential category members were unfamiliar, we did not expect the participants to have preconceived notions about their features or strong biases regarding the importance of the latter in determining oxygen resistance, and this was indeed what we observed. No feature was consistently judged as more important than the others at the beginning of the experiment. At the end of successful initial learning, we would expect the misconception feature to have accrued maximum importance, while the other features would be judged as having zero importance. In the present experiments, we refrained from eliciting importance ratings after the initial learning phase in order to avoid signaling to the participants that the target training was in any way different from the initial training. However, we did indeed observe the expected effect in past work on re-categorization (Ohlsson \& Cosejo, 2014). Finally, we would expect the importance ratings at the end of target training to assign maximum importance to the target feature, and zero importance to all other features. This pattern was not observed in any of the three experiments (nor in past work). Instead, both the initial and target features were consistently rated as more important than the other features (see Figures 3, 5, \& 6). This suggests that the participant's knowledge state after completing the target training cannot be described merely as having acquired the target category, as a cognitive conflict perspective would suggest. Instead, consistent with recent work on the persistence of misconceptions, our participants' final knowledge state included traces of both the initial and the target category definitions.

In sum, our results suggest that learners can adopt a new conception with or without cognitive conflict, but also conclude that the misconception and target attributes are important. How is this discrepancy resolved? It may be possible that some learners did not perceive direct refutation or the absence of refutation as cause to necessarily abandon their belief that the prior conception may still hold importance. Common sense would dictate that if something was once important it might become important again. For example, learners in both conditions might assume that the misconception could become important again at some later time (i.e., abeyance) and therefore, should adopt the target in the interim. Learner abeyance would avoid explicit revisions to knowledge in the absolute sense (reflected by bacteria ratings), but still produce target learning outcomes that directly or indirectly refute misconception learning in the interim (reflected by performance rate). That is, in order to reach mastery on target learning, participants must not follow the misconception rule.

A learner cannot perform better than chance by maintaining that the misconception feature is the sole cause of oxygen resistance or that both the misconception and target are causes of oxygen resistance. For example, in the Complete and Confirmatory-only conditions, if participants responded that misconception consistent bacteria were oxygen resistant and target consistent bacteria were oxygen resistant then the proportion correct would be $62.5 \%$ on a given target block. If either condition maintained the misconception, but not the target then proportion correct would be $25 \%$ on a given block. Since learner performance exceeded $62.5 \%$, we conclude that target learning performance across studies suggests that a revision in how learners represent oxygen resistance was evident (i.e., learning was non-monotonic).

In short, the evidence strongly implies that our experiments illustrate a case of successful non-monotonic category change in the absence of direct falsification or contradiction of a prior 
category. Our results are thus consistent with classroom studies that have found weak or nonexistent effects of cognitive conflict interventions, but go beyond such studies by avoiding some of the methodological difficulties noticed by Limón (2001). Categorization is a simple and wellstructured learning scenario in which the many variables that have been found to influence conceptual change in the classroom can be held constant across instructional conditions. Also, the simplicity of the categorization task enables us to construct a scenario in which the majority of the participants can successfully learn the target category, which is crucial for a rigorous interpretation of the outcome. Because learning was successful both with and without the contradictory information, the lack of effect is not due to some other infelicitous variable. Furthermore, the instruction in the complete condition consisted of repeated refutations that were too simple to go unnoticed: the participant said "yes" and the computer system said "no". It is not plausible that the participants failed to notice this contradiction. Finally, the categorization procedure allows us to collect temporally fine-grained data that track learning over time instead of being limited to pre- and posttests. The post-learning assessments were clear and straightforward. In particular, we were able to assess reliance on the initial conception and the target conception independently of each other. If the final knowledge state is a mixture of old and new knowledge, then post-tests that do not separate the two dimensions might underestimate the extent to which the target knowledge has in fact been acquired. The re-categorization paradigm reduces the impact of these methodological problems, so our results strengthen the conclusion that cognitive conflict is not necessary for non-monotonic cognitive change in the recategorization scenario.

\section{Limitations and Future Work}

The simplicity of the re-categorization paradigm is a two-edged sword. It is the source of its advantages, but also a source of limitations. The studies reported in this article demonstrate non-monotonic learning without conflict in a single, very constrained scenario that deviates in multiple ways from realistic learning scenarios. The target knowledge consisted of a single category, rather than a system of interrelated categories. The target concept was not embedded in an explanatory conceptual system that could suggest cause-effect relations. The learners were not explicitly told the target category definition; instead, it was acquired solely through discovery learning. The entire re-categorization process took less than an hour. The learners had no personal investment in the successful completion of the learning task. The initial category (the misconception) was acquired in the course of the experiment instead of through everyday experience.

In principle, each of these dimensions has the potential to impact the type of learning process that is triggered. In some cases, such an impact seems implausible. For example, consider the duration of the learning process. If non-monotonic learning can succeed in the absence of cognitive conflict over a short period of time, why would it not succeed over a longer time period? Why would a cognitive conflict become necessary with time? Similarly, if nonmonotonic learning processes can revise one categorical concept in the absence of cognitive conflict, why could it not serve to revise multiple concepts? On the other hand, it is intuitively plausible that a category with roots in everyday life is more resistant to change than a recent acquisition, and that cognitive conflict plays a role in overcoming that resistance. Issues of this sort cannot be settled through argument. The only way to identify the set of learning situations in which non-monotonic learning can be successful without conflict is to conduct empirical studies that scale up the current learning scenario along one or more of the dimensions mentioned in the previous paragraph. In future work, we intend to embed the target category in a (fictional) 
explanatory framework, expand the time frame of the learning task, and add a direct instruction component to the discovery learning procedure.

Whether non-monotonic learning without conflict operates only in a small subset of learning scenarios or scales up to a broad range of such scenarios, the theoretical question is what kind of process can account for this type of learning? What happened in the heads of our participants? An explanatory account must make clear why a person might seek or construct an alternative view even in the absence of dissatisfaction with the current view, and how the acquisition of the target knowledge can begin even before the current knowledge has been unlearned. Finally, it must specify how the two knowledge representations are related in the learner's mind at the end of successful learning, and how the conflict between them is managed in the course of thinking, problem solving, or further learning.

In past work, we have proposed a theoretical perspective that satisfies these constraints (Ohlsson, 2009). The Resubsumption Theory is based on the assumption that information encoding is ubiquitous. People spontaneously and continuously absorb information from the environment (Ohlsson, 2011, Chaps. 9-10). The result is a repertoire of intuitive theories, beliefs, schemas, and concepts, each generated in response to some stream of information in a monotonic manner. This ubiquitous encoding process is relatively unconstrained by prior knowledge and allows conflicts between alternative knowledge representations to remain latent. In the context of re-categorization, our participants acquired a new category definition even before they had any indication that they needed to do so, because the information about the relation between a particular feature and category membership was picked up, so to speak, merely because it was present in the stimulus stream. Hence, the target category definition was acquired in a monotonic manner, without any need for conflict with the previously acquired initial category. Nonmonotonic change is initiated when the person realizes that he or she has two ways to think about a phenomenon or a domain of experience. At that point, the need to act or think vis-à-vis the phenomenon or domain triggers response competition, with the alternative knowledge structures striving to control the overt response. Over time, the competition is decided on the basis of cognitive utility (Ohlsson, 2013). Even after the priority of use has been settled, both conceptions remain in long-term memory.

The current version of the resubsumption account is limited by its informal formulation. The three principles of ubiquitous encoding, latent conflict, and response competition on the basis of cognitive utility are not yet precise enough to support the derivation of quantitative predictions. In future work, we will translate those principles into a formal process model that can carry out the re-categorization process. Systematic variations of the model parameters will provide one source of information about the conditions under which non-monotonic learning can succeed without conflict.

The key feature of the resubsumption account is that the switch to a different conception is primarily driven by the advantages and strengths of the new conception, not by the problems or weaknesses of the prior conception. The learner's dissatisfaction with a prior conception is in this account replaced by his or her satisfaction with the alternative account. Conceptual change is more like responding to an opportunity than to fixing a broken chair.

There are practical educational implications that may follow from our findings. If we apply our experimental result (i.e., cognitive conflict is not a necessary component of nonmonotonic learning) to academic settings we might find that avoiding direct cognitive conflict, but offering inductive learning opportunities might promote the development of a correct scientific conception more readily than by beginning with conflict. As such, instructors might try 
introducing the new conception without drawing attention to the incorrect conception. After the target conception has been sufficiently developed (which is determined by its complexity) educators can then use tasks that offer learners the opportunity to apply the utility of the concept in a setting that would have otherwise automatically resulted in the application of the naïve conception.

For example, a student that adamantly believes that global warming is explained by normal warming and cooling cycles would likely be dismissive of a teacher that was telling him or her that he or she is wrong because of (insert copious amounts of data here). Alternatively, if an instructor instead presented specific pieces of evidence in a piecemeal fashion that does not directly refute the naïve claims then the student may be more willing to develop the scientific conception. Once the scientific evidence for global warming has reached a critical mass (i.e., its perceived utility in explaining phenomenon supersedes the naïve conception) the student can then be given an opportunity to explain. For example, what the impact of fossil fuels might be on the environment. At this point, the student may be able to naturally adopt the scientific theory without being confronted with a direct cognitive conflict because its perceived cognitive utility is greater. This type of learner environment mimics the structure of the Resubsumption Theory. By avoiding direct cognitive conflict, but being offered an opportunity to strengthen the utility for a scientific conception, a learner might be more willing to accept and apply the correct conception when finally confronted with a choice between the scientifically correct conception and naïve theory. 


\section{References}

Ariasi, N. \& Mason, L. (2014). From covert processes to overt outcomes of refutation text reading: The interplay of science text structure and working memory capacity through eye fixations. International Journal of Science and Mathematics Education, 12, 493-523.

Ashby, F. G., \& Maddox, W. T. (2005). Human category learning. Annual Review of Psychology, 56, 149-178. http://dx.doi.org/10.1146/annurev.psych.56.091103.070217

Babai, R., \& Amsterdamer, A. (2008). The persistence of "solid" and "liquid" misconceptions: A reaction time study. Journal of Science Education and Technology, 17, 553-559.

Babai, R., Sekal, R., \& Stavy, R. (2010). Persistence of the intuitive conception of living things in adolescence. Journal of Science Education and Technology, 19, 20-26.

Başer, M. (2006). Fostering conceptual change by cognitive conflict based instruction on students' understanding of heat and temperature concepts. Eurasia Journal of Mathematics, Science and Technology Education, 2, 96-113.

Berg, E. (1948). A simple objective technique for measuring flexibility in thinking. Journal of General Psychology, 39, 15-22.

Çalik, M., Kolomuç, A., \& Karagölge, Z. (2010). The effect of conceptual change pedagogy on students' conceptions of rate of reaction. Journal of Science Education and Technology, $19,422-433$.

Carey, S. (2009). The origin of concepts. New York: Oxford University Press.

Chan, C., Burtis, J., \& Bereiter, C. (1997). Knowledge building as a mediator of conflict in conceptual change. Cognition and Instruction, 1, 1-40.

Chi, M. T. H. (2005). Common sense conceptions of emergent processes: Why some misconceptions are robust. Journal of the Learning Sciences, 14, 161-199.

Chi, M. T. H. (2008). Three types of conceptual change: belief revision, mental model transformation, and categorical shift. In S. Vosniadou (Ed.), International handbook of research on conceptual change (pp. 61-82). New York: Routledge.

Chi, M. T. H., \& Brem, S. K. (2009). Contrasting Ohlsson's Resubsumption Theory with Chi's Categorical Shift Theory. Educational Psychologist, 44, 58-63. http://dx.doi.org/10.1080/00461520802616283

Chi, M.T.H. \& Hausmann, R.G.M. (2003). Do radical discoveries require ontological shifts? In L.V. Shavinina (Ed.), International handbook on innovation (vol. 3, pp. 430-444). Oxford, UK: Elsevier.

Chi, M.T.H., Slotta, J.D. \& de Leeuw, N. (1994). From things to processes: A theory of conceptual change for learning science concepts. Learning and Instruction, 4, 27-43.

Chi, M.T.H., \& Roscoe, R.D. (2002). The processes and challenges of conceptual change. In M. Limón and L. Mason (Eds.), Reconsidering conceptual change: Issues in theory and practice (pp. 3-27). Dordrecht, The Netherlands: Kluwer.

Chia, A. (1998). Seeing and believing: The variety of scientists' responses to contrary data. Science Communication, 19, 366-391.

Chinn, C.A., \& Brewer, W.F. (1993). The role of anomalous data in knowledge acquisition: A theoretical framework and implications for science instruction. Review of Educational Research, 63, 1-49.

Clark, D. B. (2006). Longitudinal conceptual change in students' understanding of thermal equilibrium: An examination of the process of conceptual restructuring. Cognition and Instruction, 24, 467-563.

Cordova, J., Sinatra, G., Jones, S., Taatsoobshirazi, G. \& Lombardi, D. (2014). Confidence in 
prior knowledge, self-efficacy, interest and prior knowledge: Influences on conceptual change. Contemporary Educational Psychology, 39, 164-174.

Cosejo, D. G., Oesterreich, J., \& Ohlsson, S. (2009). Re-categorization: Restructuring in categorization In N.A. Taatgen \& H. van Rijn (Eds.), Proceedings of the 31th Annual Conference of the Cognitive Science Society. Austin, TX: Cognitive Science Society.

Dega, B. G., Kriek, J., \& Mogese, T. F. (2013). Students' conceptual change in electricity and magnetism using simulations: A comparison of cognitive perturbation and cognitive conflict. Journal of Research in Science Teaching, 50, 677-698.

DiSessa, A. A. (1993). Towards an epistemology of physics. Cognition and Instruction, 10, $105-$ 225.

DiSessa, A., \& Sherin, B. (1998). What changes in conceptual change? International Journal of Science Education, 20, 1155-1191.

DiSessa, A., Gillespie, N., \& Easterly, J. (2004). Coherence versus fragmentation in the development of the concept of force. Cognitive Science, 28, 843-900. DOI 10.007/s11191-013-9658-6.

Doumas, L., \& Hummel, J. (2013). Comparison and mapping facilitate relation discovery and predication. PLOS One, 8(6): e63889. doi:10.1371/ journal.pone.0063889

Ecker, U., Lewandowsky, S., Swire, B., \& Chang, D. (2011). Correcting false information in memory: Manipulating the strength of misinformation encoding and its retraction. Psychonomic Bulletin \& Review, 18, 570-578.

Gopnik, A., \& Meltzoff, A. N. (1997). Words, thoughts, and theories. Cambridge MA: MIT Press.

Gopnik, A., \& Wellman, H. M. (1994). The theory theory. In L.A. Hirschfeld and S.A. Gelman (Eds.), Mapping the mind: Domain specificity in cognition and culture (pp. 255-293). Cambridge, UK: Cambridge University Press.

Harmon-Jones, E., \& Mills, J. (1999). Cognitive dissonance: Progress on a pivotal theory in social psychology. Washington, D.C.: American Psychological Association.

Heddy, B., \& Sinatra,G. (2013). Transforming misconceptions: Using transformative experience to promote positive affect and conceptual change in students learning about biological evolution. Science Education, 97, 723-744.

Hewson, P.W. \& Hewson, M.G.A. (1984). The role of conceptual conflict in conceptual change and the design of science instruction. Instructional Science, 13, pp. 1-13.

Kang, H., Scharmann, L. C., Kang, S., \& Noh, T. (2010). Cognitive conflict and situational interest as factors influencing conceptual change. International Journal of Environmental \& Science Education, 5, 383-405.

Kendler, H., \& D’Amato, M. (1955). A comparison of reversal shifts and nonreversal shifts in human concept formation behavior. Journal of Experimental Psychology, 49, 165-174.

Kitcher, P. (1993). The advancement of science. New York: Oxford University Press.

Kuhn, T. S. (1970). The structure of scientific revolutions $\left(2^{\text {nd }}\right.$ ed.). Chicago, IL: University of Chicago Press.

Lakatos, I. (1980). Philosophical papers (vol. 1): The methodology of scientific research programmes). Cambridge, UK: Cambridge University Press.

Lee, G., \& Byun, T. (2012). An explanation for the difficulty of leading conceptual change using a counterintuitive demonstration: The relationship between cognitive conflict and response. Research in Science Education, 42, 943-965.

Limón, M. (2001). On the cognitive conflict as an instructional strategy for conceptual change: a 
critical appraisal. Learning and Instruction, 11, 357-380.

Lin, J.-Y. (2007). Responses to anomalous data obtained from repeatable experiments in the laboratory. Journal of Research in Science Teaching, 44, 506-528.

Maio, G., \& Haddock, G. (2007). Attitude change. In A. W. Kruglanski and E. T. Higgins (Eds.), Social psychology: Handbook of basic principles $\left(2^{\text {nd }}\right.$ ed., pp. 565-586). New York, NY: Academic Press.

Nersessian, N. J. (2008). Creating scientific concepts. Cambridge MA: MIT Press.

Nickerson, R. (1998). Confirmation bias: A ubiquitous phenomenon in many guises. Review of General Psychology, 2, 175-220.

Ohlsson, S. (2009). Resubsumption: A possible mechanism for conceptual change and belief revision. Educational Psychologist, 44, 20- 40. http://dx.doi.org/10.1080/00461520802616267

Ohlsson, S. (2011). Deep learning: How the mind overrides experience. Cambridge, UK: Cambridge University Press. http://dx.doi.org/10.1017/CBO9780511780295

Ohlsson, S. (2013). Beyond evidence-based belief formation: How normative ideas have constrained conceptual change research. Frontline Learning Research, 2, 0-85.

Ohlsson, S., \& Cosejo, D. G. (2014). What can be learned from a laboratory model of conceptual change? Descriptive findings and methodological issues. Science \& Education, 23, 14851504.

Özdemir, G., \& Clark, D. B. (2007). An overview of conceptual change theories. Eurasia Journal of Mathematics, Science \& Technology Education, 3, 351-361.

Piaget, J. (1985/1975). The equilibration of cognitive structures: The central problem of intellectual development (transl. T. Brown and K. J. Thampy). Chicago, IL: University of Chicago Press.

Popper, K. (1959/1972). The logic of scientific discovery (translation by Ohlsson, revised version). London, UK: Hutchinson.

Posner, G., Strike, K., Hewson, P., \& Gertzog, W. (1982). Accommodation of a scientific conception: Toward a theory of conceptual change. Science Education, 66, 211-227.

Potvin, P., Masson, S., LaFortune, S., \& Cyr, G. (2015). Persistence of the intuitive conception that heavier objects sink more: A reaction time stud with different levels of interference. International Journal of Science and Mathematics Education, 13, 21-43.

Sewell, D., \& Lewandowsky, S. (2011). Restructuring partitioned knowledge: The role of recoordination in category learning. Cognitive Psychology, 62, 81-122.

Shtulman, A., \& Valcarcel, J. (2012). Scientific knowledge suppresses but does not supplant earlier intuitions. Cognition, 124, 209-215.

Sinatra, G., \& Broughton, S. (2011). Bridging reading comprehension and conceptual change in science education: The promise of refutation text. Reading Research Quarterly, 46, 374393.

Sinatra, G., \& Pintrich, P., (Eds.), (2003). Intentional conceptual change. Mahwah, NJ: Erlbaum.

Slotta, J. D., \& Chi, M. T. H. (2006). Helping students understand challenging topics in science through ontology training. Cognition and Instruction, 24, 261-289.

Slotta, J. D., Chi, M.T.H. \& Joram, E. (1995). Assessing students' misclassifications of physics concepts: An ontological basis for conceptual change. Cognition and Instruction, 13, 373-400.

Strike, K.A., \& Posner, G.J. (1982). Conceptual change and science teaching. European Journal of Science Education, 4, 231-240. 
Strike, K.A., \& Posner, G.J. (1992). A revisionist theory of conceptual change. In R. Duschl \& R. Hamilton (eds.), Philosophy of science, cognitive psychology, and educational theory and practice (pp. 147-176). Albany, NY: SUNY Press.

Taylor, E., \& Ahn, W.-k. (2012). Causal imprinting in causal structure learning. Cognitive Psychology, 65, $381-413$.

Thagard, P, (1992). Conceptual revolutions. Princeton, NJ: Princeton University Press.

Toulmin, S. (1972). Human understanding. London, UK: Oxford University Press.

Trevors, G., \& Muis, K. (2014). Effects of text structure, reading goals and epistemic beliefs on conceptual change. Journal of Research in Reading, 00, 1-26.

Vosniadou, S. (1994). Capturing and modeling the process of conceptual change. Learning and Instruction, 4, 45-69.

Vosniadou, S., \& Brewer, W.F. (1992). Mental models of the earth: A study of conceptual change in childhood. Cognitive Psychology, 24, 535-585.

Vosniadou, S., \& Skopeliti, I. (2014). Conceptual change from the framework theory side of the fence. Science \& Education, 23(7), 1427-1445. DOI 10.1007/s11191-013-9640-3.

Walsh, C., \& Johnson-Laird, P. (2009). Changing your mind. Memory \& Cognition, 37, 624-631.

Zohar, A., \& Aharon-Kravetsky, S. (2005). Exploring the effects of cognitive conflict and direct teaching for students of different academic levels. Journal of Research in Science Teaching, 42, 829-855. 
Table 1

The means and (standard deviations) for percentage correct for blocks 6-10.

\begin{tabular}{lccccc}
\hline & Block & Block & Block & Block & Block \\
& 6 & 7 & 8 & 9 & 10 \\
\hline Complete & 40.63 & 64.64 & 72.86 & 81.58 & 86.06 \\
& $(16.61)$ & $(27.43)$ & $(28.11)$ & $(24.49)$ & $(19.89)$ \\
Confirmation & 63.72 & 77.78 & 83.51 & 88.54 & 88.02 \\
& $(16.89)$ & $(19.33)$ & $(20.27)$ & $(16.06)$ & $(20.51)$ \\
\hline
\end{tabular}


Table 2

The means and (standard deviations) for percentage correct for blocks 6-10.

\begin{tabular}{lrrrrr}
\hline & Block & Block & Block & Block & Block \\
& \multicolumn{1}{c}{6} & \multicolumn{1}{c}{7} & \multicolumn{1}{c}{8} & \multicolumn{1}{c}{9} & \multicolumn{1}{c}{10} \\
\hline Complete & 44.94 & 68.75 & 78.87 & 80.06 & 83.93 \\
& $(20.31)$ & $(30.94)$ & $(29.01)$ & $(28.55)$ & $(26.34)$ \\
Confirmation & 68.75 & 82.14 & 78.87 & 85.42 & 85.12 \\
& $(12.66)$ & $(19.89)$ & $(16.11)$ & $(19.8)$ & $(23.67)$ \\
\hline
\end{tabular}


Table 3

The means and (standard deviations) for percentage correct for blocks 6-10.

\begin{tabular}{lcrrrr}
\hline & Block & Block & Block & Block & Block \\
& \multicolumn{1}{c}{6} & \multicolumn{1}{c}{7} & \multicolumn{1}{c}{8} & \multicolumn{1}{c}{9} & \multicolumn{1}{c}{10} \\
\hline Complete & 57.23 & 84.77 & 87.11 & 91.01 & 89.06 \\
& $(17.69)$ & $(17.96)$ & $(17.74)$ & $(15.38)$ & $(19.31)$ \\
Confirmation & 89.06 & 95.18 & 92.76 & 93.04 & 84.09 \\
& $(8.64)$ & $(7.36)$ & $(14.88)$ & $(12.73)$ & $(14.17)$ \\
\hline
\end{tabular}




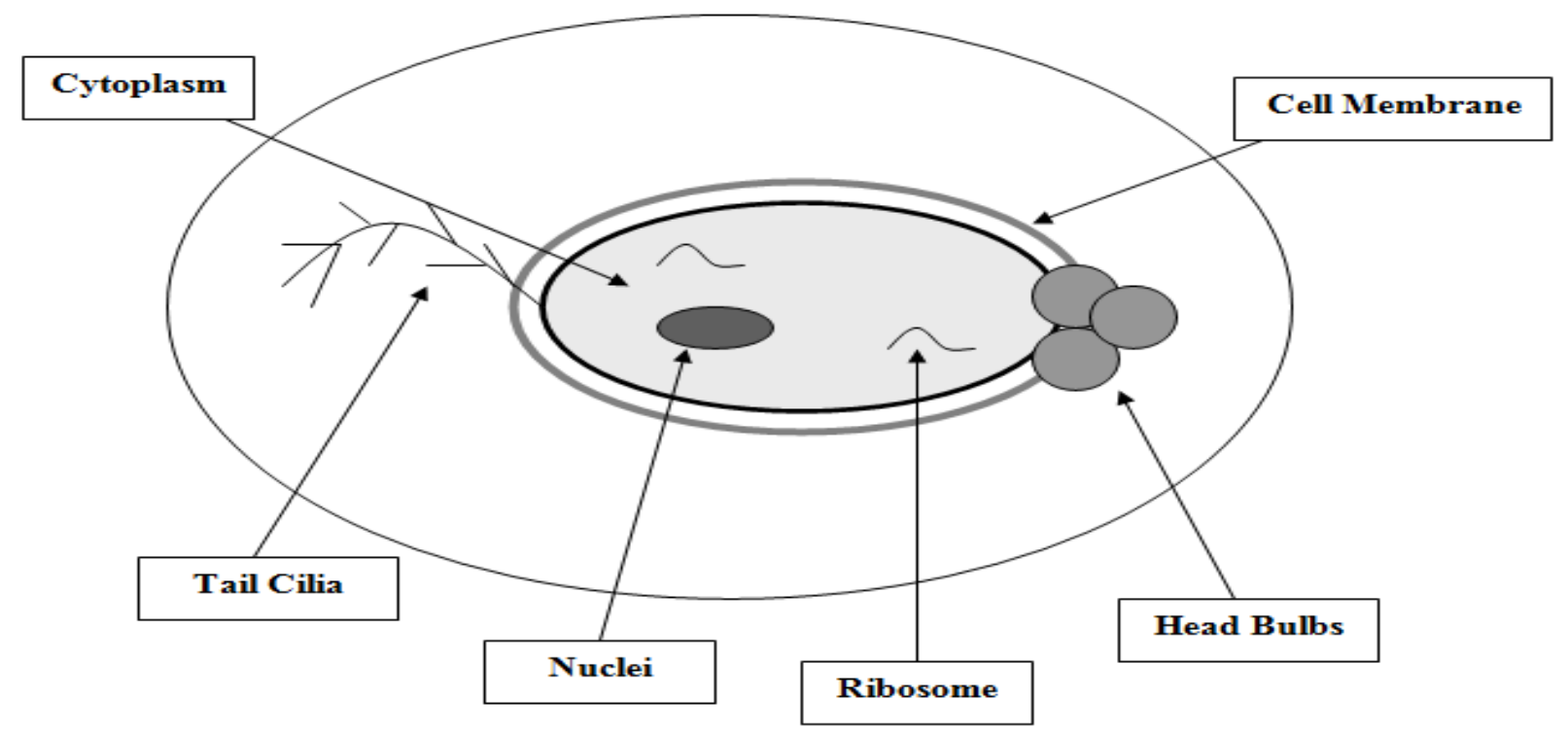

Figure 1. Example bacterium with parts labeled. 

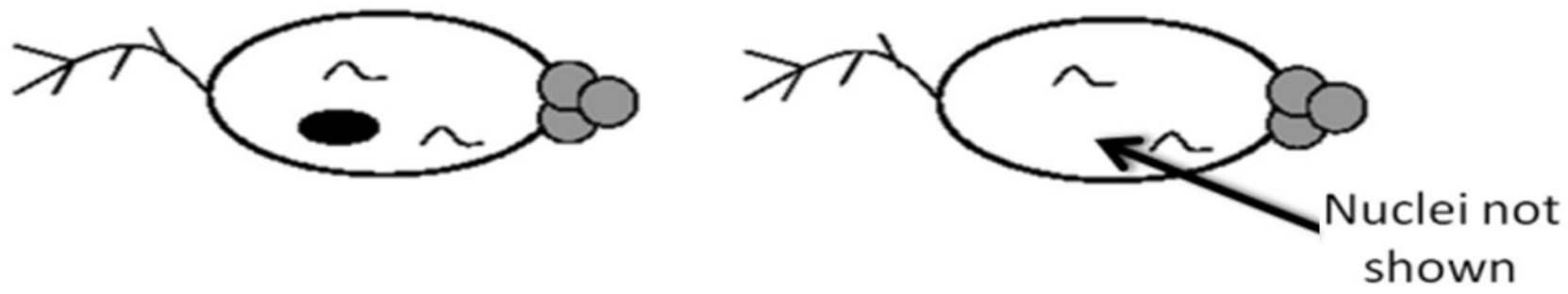

Figure 2. Bacteria with and without nuclei shown. 


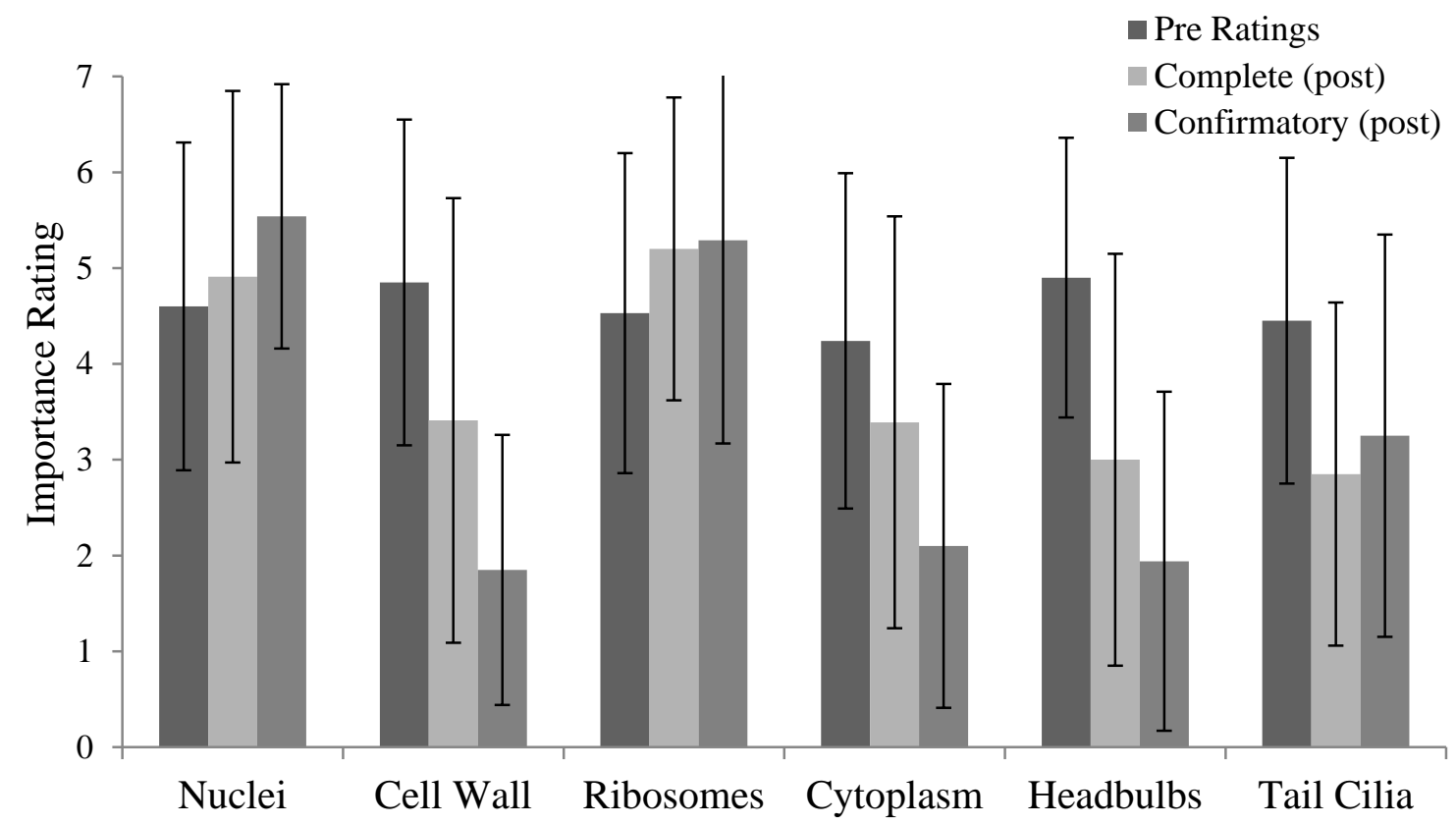

Figure 3. Experiment 1 importance ratings for bacteria attributes pre and post (error bars denote one standard deviation). 


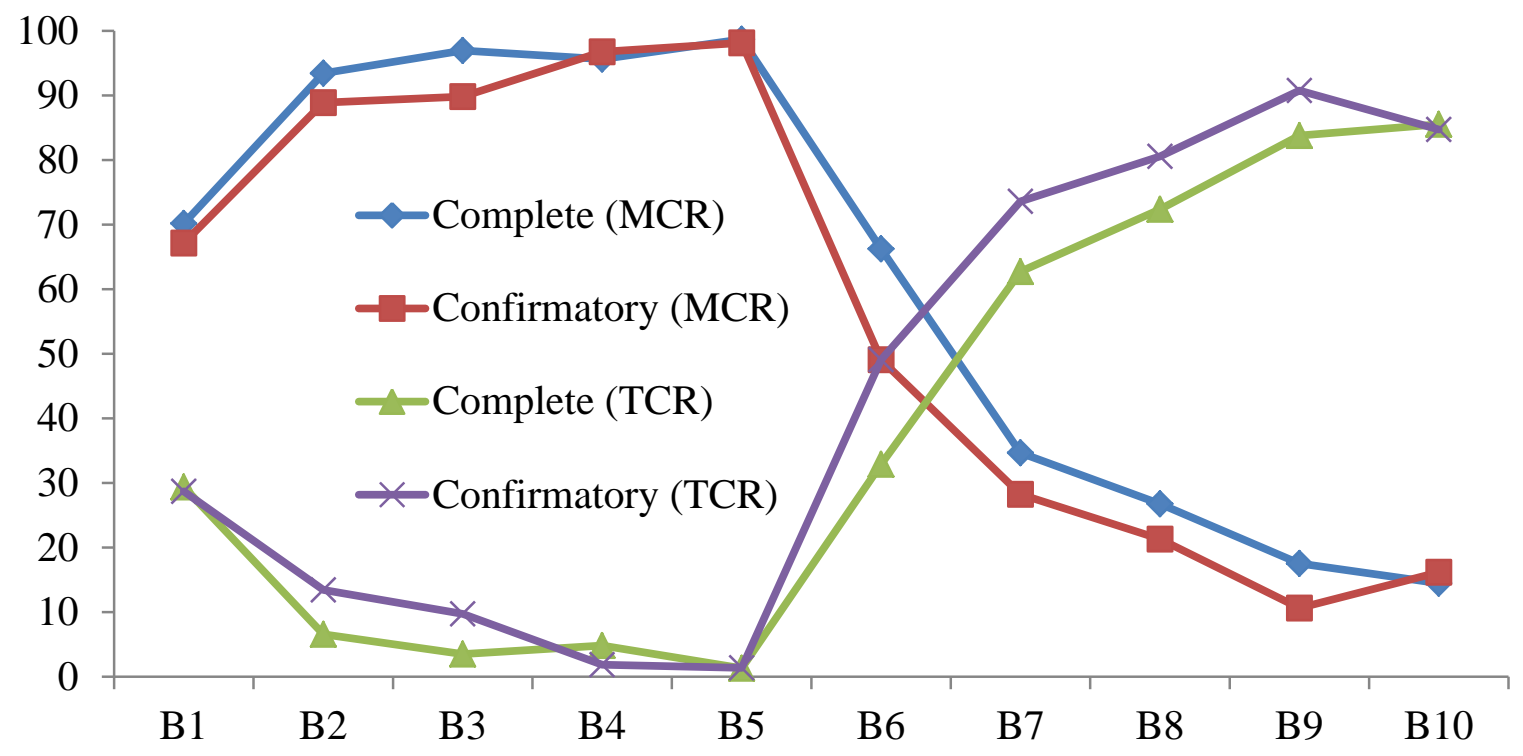

Figure 4. Percentage consistent with response type by condition. 


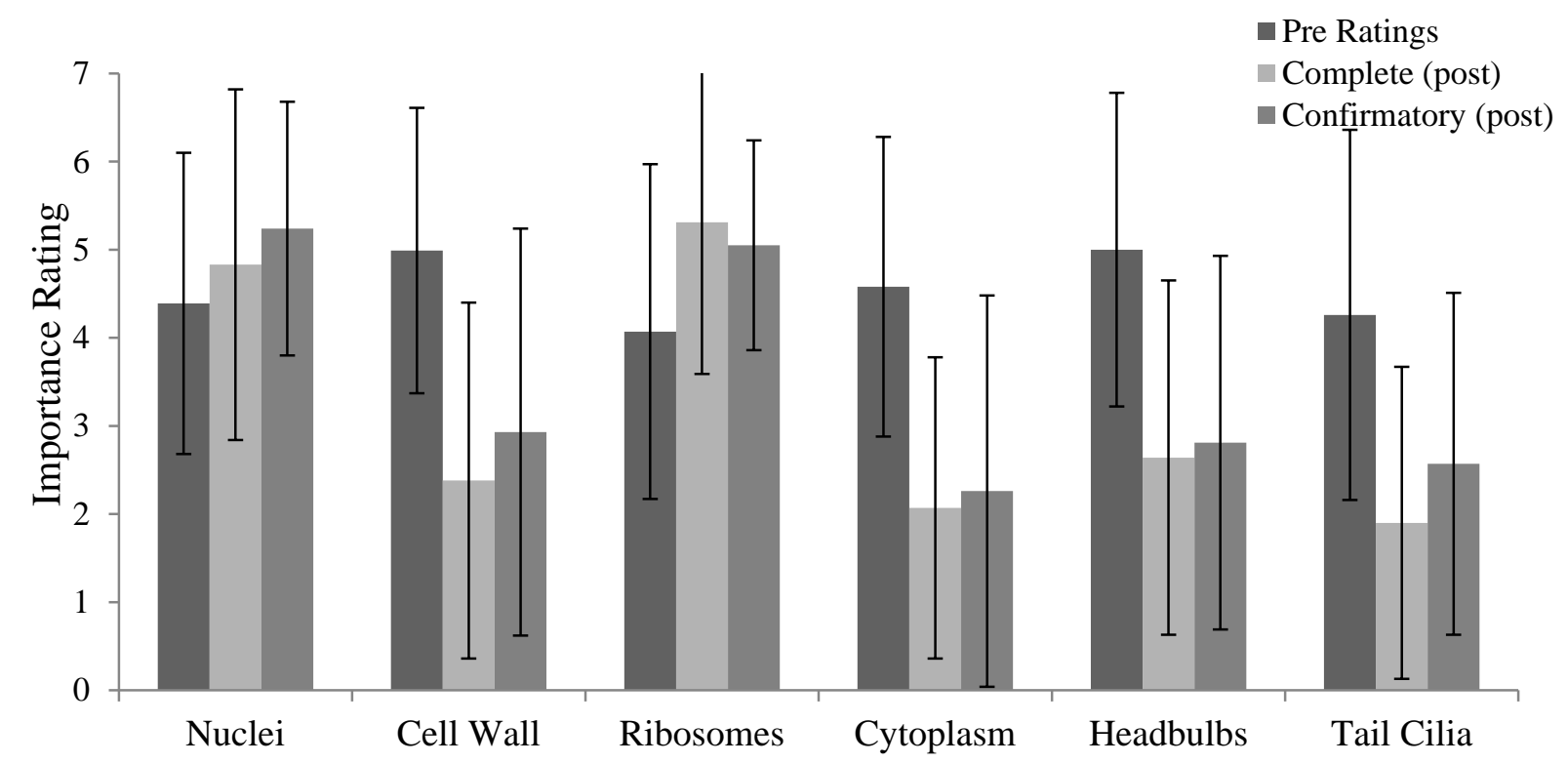

Figure 5. Experiment 2 importance ratings for bacteria attributes pre and post (error bars denote one standard deviation). 


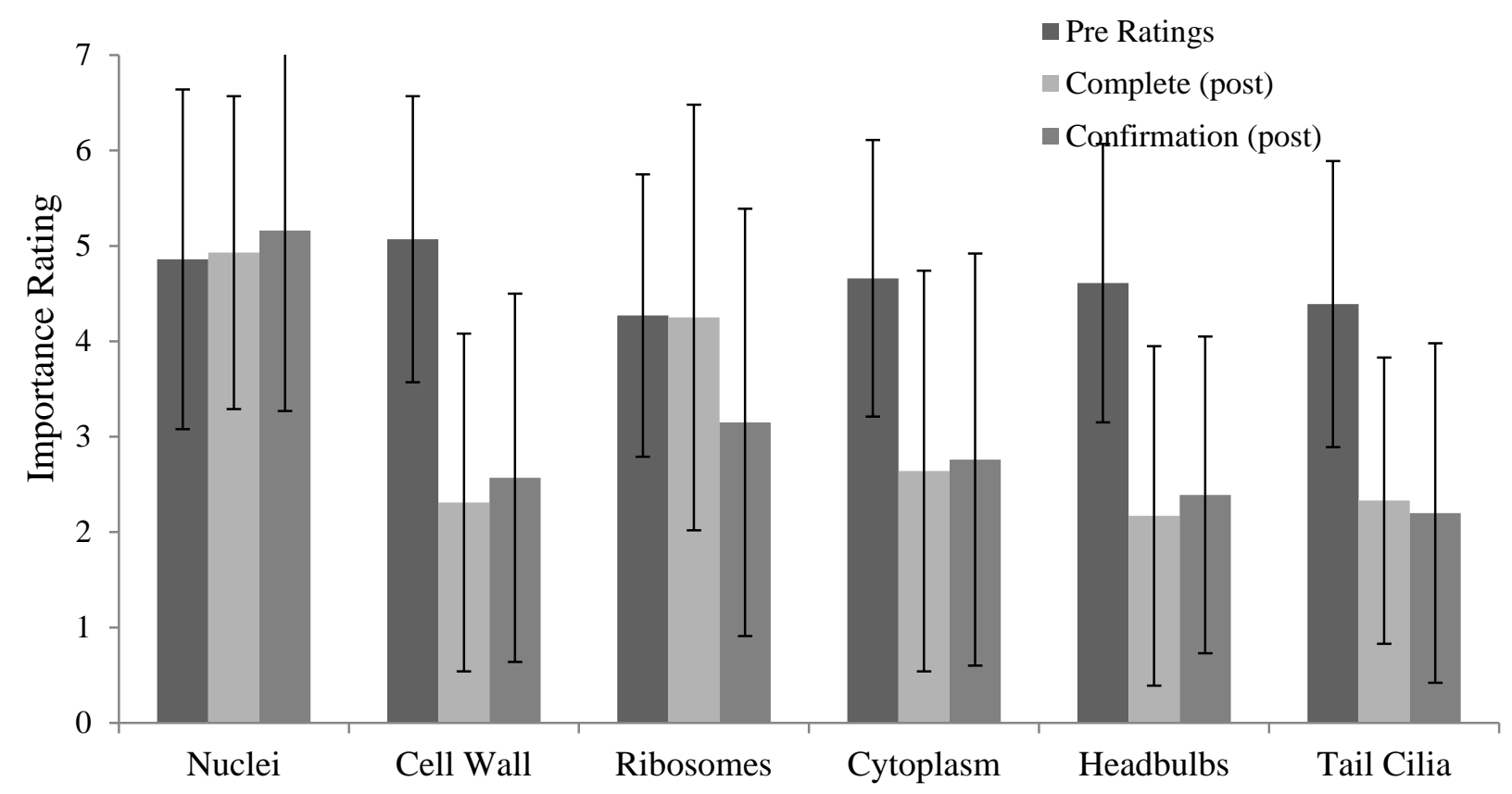

Figure 6. Experiment 3 importance ratings for bacteria attributes pre and post (error bars denote one standard deviation). 